Article

\title{
Investigating the Role of Shell Thickness and Field Cooling on Saturation Magnetization and Its Temperature Dependence in $\mathrm{Fe}_{3} \mathrm{O}_{4} / \gamma-\mathrm{Fe}_{2} \mathrm{O}_{3}$ Core/Shell Nanoparticles
}

\author{
Ihab M. Obaidat ${ }^{1, *}$, Chiranjib Nayek ${ }^{1}$ and Kaustuv Manna ${ }^{2}$ \\ 1 Department of Physics, United Arab Emirates University, Al-Ain 15551, UAE; chiranjibnayek@gmail.com \\ 2 Max-Planck-Institute for Chemical Physics of Solids, Nöthnitzer Straße-40, 01187 Dresden, Germany; \\ kaustuvmanna@gmail.com \\ * Correspondence: iobaidat@uaeu.ac.ae; Tel.: +971-(3)-7136321; Fax: +971-(3)-7136944
}

Received: 31 October 2017; Accepted: 21 November 2017; Published: 5 December 2017

\begin{abstract}
Understanding saturation magnetization and its behavior with particle size and temperature are essential for medical applications such magnetic hyperthermia. We report the effect of shell thickness and field cooling on the saturation magnetization and its behavior with temperature in $\mathrm{Fe}_{3} \mathrm{O}_{4} / \gamma-\mathrm{Fe}_{2} \mathrm{O}_{3}$ core/shell nanoparticles of fixed core diameter $(8 \mathrm{~nm})$ and several shell thicknesses. $\mathrm{X}$-ray diffraction (XRD) analysis and transmission electron microscopy (TEM, high-resolution transmission electron microscopy (HRTEM)) were used to investigate the phase and the morphology of the samples. Selected area electron diffraction (SAED) confirmed the core/shell structure and phases. Using a SQUID (San Diego, CA, USA), magnetic measurements were conducted in the temperature range of 2 to $300 \mathrm{~K}$ both under zero field-cooling (ZFC) and field-cooling (FC) protocols at several field-cooling values. In the ZFC state, considerable enhancement of saturation magnetization was obtained with the increase of shell thickness. After field cooling, we observed a drastic enhancement of the saturation magnetization in one sample up to $120 \mathrm{emu} / \mathrm{g}$ (50\% larger than the bulk value). In both the FC and ZFC states, considerable deviations from the original Bloch's law were observed. These results are discussed and attributed to the existence of interface spin-glass clusters which are modified by the changes in the shell thickness and the field-cooling.
\end{abstract}

Keywords: nanoparticles; magnetization; shell thickness; Bloch's law

\section{Introduction}

Magnetic nanoparticles have drawn large interest due to their applications in the field of magnetic recording and other areas [1,2]. The unique magnetic behaviour exposed these materials to a very wide range of medical applications such as drug delivery systems [3], magnetic resonance imaging (MRI) [4-7], and for cancer therapy [8]. Due to several factors [5,6], the superparamagnetic iron oxide nanoparticles (SPIONs) such as magnetite $\left(\mathrm{Fe}_{3} \mathrm{O}_{4}\right)$ and maghemite $\left(\gamma-\mathrm{Fe}_{2} \mathrm{O}_{3}\right)$ are being studied extensively for medical field applications. The magnetic nanoparticles exhibit interesting magnetic behavior due to finite size effects such as high surface-to-volume ratio, and different crystal structures. When the size of the particle decreases, the surface to volume ratio increases causing a large portion of the atoms to be at the surface compared to those in the core of the particle. This large surface to volume ratio changes the physical, chemical, and mechanical properties compared to those of the bulk materials. The magnetic nanoparticles are assumed to be a single domain particle with uniaxial anisotropy where the orientation of its magnetic moment points "up" or "down" in a zero field along the easy axis. Below a critical size, the magnetic nanoparticles become a single magnetic domain and retain a superparamagnetic behavior as long as the temperature is above the blocking temperature. In the 
superparamagnetic state, the magnetization vectors of the nanoparticles fluctuate along the easy axis of magnetization, inducing a large magnetic moment in each nanoparticle. These magnetic moments continuously change their orientations. When a magnetic field is applied externally, superparamagnetic particles exhibit a fast response to changes of magnetic field with zero remnant magnetization and zero coercivity. Thus, in the superparamagnetic region, a magnetic nanoparticle behaves as a large paramagnetic atom. In case of a temperature below the blocking temperature $\left(T_{\mathrm{B}}\right)$, the thermal agitation will not be large enough to cause fluctuations in the magnetic moments of the nanoparticles where they freeze in random orientations very similar to the ferromagnetic state. However, the surface atoms experience a different environment than those on the core of the particle. The surface magnetization is controlled by several parameters such as atomic vacancies, change in atomic coordination, dangling bonds, and lattice disorder [9-21]. These parameters result in disordered surface spins. The magnetic nanoparticles assembly exhibit hysteresis for $T<T_{\mathrm{B}}$ and a non-hysteric behavior for $T>T_{\mathrm{B}}$. The magnetic behavior for the monodispersed and non-interacting single domain magnetic particles can be explained on the basis of Néel relaxation and the Bean-Livingston approaches. Saturation magnetization $\left(M_{\mathrm{S}}\right)$, decreases with the cube root of temperature, which is called Bloch's law.

One important application of magnetic nanoparticles is magnetic hyperthermia, where the living organ can be heated to a temperature large enough to destroy tumor cells while not affecting healthy ones [6]. This heating process can be achieved by inserting magnetic nanoparticles in the cells and exposing them to an alternating magnetic field. The amount of heat generated by heating the nanoparticles depends on several factors [6]. Most models that are used to understand the heating process are based on Rosensweig's theory [22] where, as an approximation, the saturation magnetization is considered to be constant with temperature. However, it is well-established that the saturation magnetization of nanoparticles changes significantly with temperature [23-28].

Core/shell nanoparticles can provide a mechanism of tuning the magnetization of nanoparticles by controlling the core and shell sizes and phases. The temperature dependence of magnetization can also be influenced by the atomic nature of the core/shell interface where interface magnetic anisotropy is introduced and may vary the total magnetic anisotropy of the particle significantly [6]. For core/shell nanoparticles, magnetic properties are very interesting. The magnetic properties of such a core/shell system can be tuned by changing the shell material and dimensions. Recently, tremendous efforts have paved the way to control magnetic properties by varying the core/shell phase compositions, shape, and dimensions [29-35]. Though, there are reports regarding the magnetic effect in different core/shell systems, the effect of shell thickness and field cooling on $M_{\mathrm{S}}$ and its temperature dependence, $M_{\mathrm{S}}(T)$ in ferrimagnetic/ferrimagnetic core/shell system was not reported (in our understanding). In this work, we have conducted detailed magnetic measurements on $\mathrm{Fe}_{3} \mathrm{O}_{4} / \gamma-\mathrm{Fe}_{2} \mathrm{O}_{3}$ core/shell nanoparticles of fixed core diameter and variable shell thickness. The core diameter of all samples is $8 \mathrm{~nm}$, while the shell thickness was varied from 1 to $5 \mathrm{~nm}$. We investigated the effect of shell thicknesses and field cooling on $M_{S}$ and $M_{S}(T)$.

\section{Materials and Methods}

We have synthesized iron oxide $\mathrm{Fe}_{3} \mathrm{O}_{4} / \gamma-\mathrm{Fe}_{2} \mathrm{O}_{3}$ core/shell nanoparticles through the usual co-precipitation method. The $\mathrm{FeCl}_{3} \cdot 6 \mathrm{H}_{2} \mathrm{O}(0.1 \mathrm{M})$ and $\mathrm{FeCl}_{2} \cdot 4 \mathrm{H}_{2} \mathrm{O}(0.05 \mathrm{M})$ were used as precursor materials. The precursor materials were dissolved in deionized water separately and mixed at room temperature after which ammonium hydroxide solution (25\%) was slowly added until PH reached 10. The mixed solution was kept at $80^{\circ} \mathrm{C}$ and the reaction was made to occur in atmospheric pressure. The reaction time was varied between 1 and $3 \mathrm{~h}$ to get different size nanoparticles (reaction times of 1,2 and $3 \mathrm{~h}$ resulted in shell thicknesses of 1,3 and $5 \mathrm{~nm}$, respectively). The final precipitation was collected and dried at $120^{\circ} \mathrm{C}$ in vacuum. There are two main parameters in our synthesis method, (1) the reaction time and (2) the diffusion time. The reaction time was varied to obtain different initial particle diameters. The atomic oxygen plays a major role inducing $\gamma-\mathrm{Fe}_{2} \mathrm{O}_{3}$ phase. This is due to the direct logarithmic kinetic diffusion with a logarithmic relation between $\gamma-\mathrm{Fe}_{2} \mathrm{O}_{3}$ thicknesses versus 
oxygen exposure time [36]. The diffusion was allowed to occur in an ambient atmosphere (in the presence of oxygen). In this way, the reaction mechanism which involves inward anionic diffusion is responsible for the occurrence of $\gamma-\mathrm{Fe}_{2} \mathrm{O}_{3}$ phase at the core surface. The diffusion time was varied to obtain different $\gamma-\mathrm{Fe}_{2} \mathrm{O}_{3}$ shell thicknesses. By trial and error, we have optimized these two parameters to obtain fixed core and different shell thicknesses. The larger the reaction time, the larger is the initial particle size. The larger the oxidation (diffusion) time, the larger is the oxidized layer (shell thickness). Thus, to obtain larger/smaller shell thickness, we started with larger/smaller particles (longer/shorter reaction times) and allowed for longer/shorter diffusion times. By optimizing these two parameters we were able to determine the suitable initial particle size for each diffusion time such that we obtained a fixed core for each case [37]. We found that when the reaction time is $1 \mathrm{~h}$ and the diffusion time is $1 \mathrm{~h}$ the core diameter is $8 \mathrm{~nm}$ and the shell thickness is $1 \mathrm{~nm}$. When the reaction time is $2 \mathrm{~h}$ and the diffusion time is $2 \mathrm{~h}$, the core diameter is $8 \mathrm{~nm}$ and the shell thickness is $3 \mathrm{~nm}$. When the reaction time is $3 \mathrm{~h}$ and the diffusion time is $3 \mathrm{~h}$, the core diameter is $8 \mathrm{~nm}$ and the shell thickness is $5 \mathrm{~nm}$.

The X-ray diffraction (XRD) analysis was conducted using a PANalytical X'Pert Pro (Almelo, The Netherlands) X-ray diffractometer. The morphology of the nanoparticles were confirmed by high resolution transmission electron microscopy (HRTEM), and selected area electron diffraction (SAED). The transmission electron microscopy (TEM) measurements were performed using an FEI, TECNAI G ${ }^{2}$ F30, S-TWIN (GG Eindhoven, The Netherlands) microscope operating at $300 \mathrm{kV}$ equipped with a GATAN Orius SC1000B CCD camera (GG Eindhoven, The Netherlands). A SQUID (San Diego, CA, USA) from Quantum Design, was used to conduct the magnetic measurements. Magnetic moment versus applied magnetic field up to $\pm 3 \mathrm{~T}$ was measured for all the samples at several temperatures $(2,10,30,50,70$ and $100 \mathrm{~K})$ after cooling the samples in the zero-field cooled (ZFC) and field-cooled (FC) states. The samples with shell thicknesses of 1,3 and $5 \mathrm{~nm}$ are named as S1, S2 and S3, respectively.

\section{Results and Discussion}

\subsection{Structural and Morphological Analysis}

The phase purity of the $\mathrm{Fe}_{3} \mathrm{O}_{4} / \gamma-\mathrm{Fe}_{2} \mathrm{O}_{3}$ core/shell nanoparticle samples was confirmed by XRD analysis. The pattern is presented for $2 \theta$ ranging from $20^{\circ}$ to $70^{\circ}$ along with all the peaks indexed according to the $\mathrm{Fe}_{3} \mathrm{O}_{4}$ core and shown in Figure 1a. We did not observe any signature of secondary phases, which confirms the phase purity of our samples. The XRD peak due to (311) plane for both $\mathrm{Fe}_{3} \mathrm{O}_{4}$ and $\gamma-\mathrm{Fe}_{2} \mathrm{O}_{3}$ phases were found to overlap with each other whereas the peaks due to (511), (422), (311), (220) and (440) lattice planes corresponding to $\mathrm{Fe}_{3} \mathrm{O}_{4}$ core phase were clearly visible.

The particles in all samples were observed to be almost spherical in shape. Figure 2 displays the TEM images and the particle size histograms of the three samples. The particle size histograms were obtained from TEM measurements on many particles. Some aggregations can be observed in the TEM images. The average particle sizes calculated from the TEM images for samples S1, S2 and S3 samples were found to be $9 \mathrm{~nm}, 11 \mathrm{~nm}$ and $13 \mathrm{~nm}$, respectively. The particle sizes were also obtained from the XRD patterns by using Scherrer's formula; $D_{\mathrm{P}}=\frac{0.94 \lambda}{\beta \cos \theta}$, where $D_{\mathrm{P}}$ is the average crystallite size, $\lambda$ is the $X$-ray wavelength, $\beta$ is the full width of half maximum (FWHM) of the XRD line, and $\theta$ is the Brag's angle. The average particle sizes calculated from Scherrer's formula were found to be $9 \mathrm{~nm}, 11 \mathrm{~nm}$ and $13 \mathrm{~nm}$, for samples S1, S2 and S3, respectively, which exactly match the average particle sizes obtained from the TEM measurements. The average core and shell dimensions were obtained from several HRTEM images. The core diameters were found to be approximately $8 \mathrm{~nm}$ for all the samples. In addition to XRD analysis, the structure and morphology of the nanoparticles were investigated using several microscopy tools such as high resolution transmission electron microscopy (HRTEM), and selected area electron diffraction (SAED). The average shell thicknesses for samples, S1, S2 and S3 were found to be 1,3 and $5 \mathrm{~nm}$, respectively. Figure $1 \mathrm{~b}$ displays the HRTEM and SAED images of sample S2. Similar HRTEM images were obtained for the other samples as displayed in Figure 1c,d. In the HRTEM images, the core appeared darker than the shell and thus displaying the 
core/shell structure of the nanoparticles. The high crystalline nature of the cores of the nanoparticles are clearly observed in Figure $1 \mathrm{~b}$. The $\mathrm{Fe}_{3} \mathrm{O}_{4} / \gamma-\mathrm{Fe}_{2} \mathrm{O}_{3}$ core/shell structure of the nanoparticles were confirmed from the selected area electron diffraction (SAED) patterns. A representative of the SAED patterns is shown in Figure 1e for sample S2. The ring type pattern shown in Figure 1e ascertains the polycrystalline nature of the sample is also confirmed by this figure. The presence of $\mathrm{Fe}_{3} \mathrm{O}_{4}$ and $\gamma-\mathrm{Fe}_{2} \mathrm{O}_{3}$ crystal planes forming the core/shell structure. The highest intensity peaks (311) for both $\mathrm{Fe}_{3} \mathrm{O}_{4}$ and $\gamma-\mathrm{Fe}_{2} \mathrm{O}_{3}$ phases were observed for $d=0.253 \mathrm{~nm}$ and $0.252 \mathrm{~nm}$, respectively. This indicates that the (311) plane observed in Figure 1e corresponds to the superimposed planes of both the phases. The (511), (422), (311) and (220) planes of $\mathrm{Fe}_{3} \mathrm{O}_{4}$ corresponding to $d=0.161,0.171,0.253$ and $0.296 \mathrm{~nm}$, respectively, in addition to the (321) and (221) planes of $\gamma-\mathrm{Fe}_{2} \mathrm{O}_{3}$ corresponding to $d=0.253 \mathrm{~nm}$ and $0.278 \mathrm{~nm}$, respectively, are all present in Figure 1e. This clearly confirms that our core/shell nanoparticles are $\mathrm{Fe}_{3} \mathrm{O}_{4} / \gamma-\mathrm{Fe}_{2} \mathrm{O}_{3}$ nanoparticles [37].
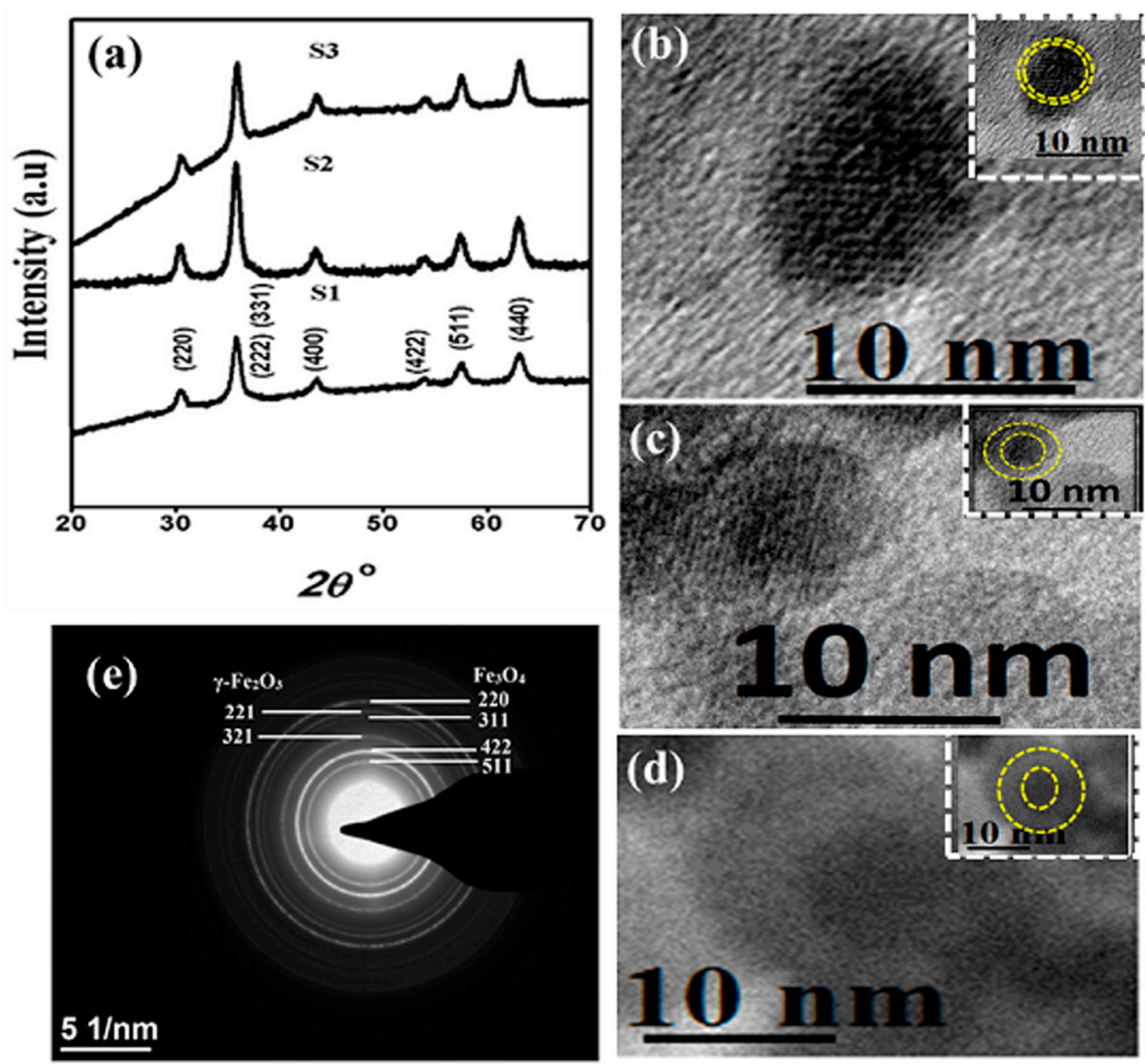

Figure 1. (a) The X-ray diffraction (XRD) patterns of $\mathrm{Fe}_{3} \mathrm{O}_{4} / \gamma-\mathrm{Fe}_{2} \mathrm{O}_{3}$ core/shell nanoparticles for all samples; (b) the high-resolution transmission electron microscopy (HRTEM) image of sample S1; (c) the HRTEM image of sample S2 (d) the HRTEM image of sample S3; (e) selected area electron diffraction (SAED) images of sample S2. (Reproduced from [37]. Copyright Obaidat, I.M., et al., 2017.). 

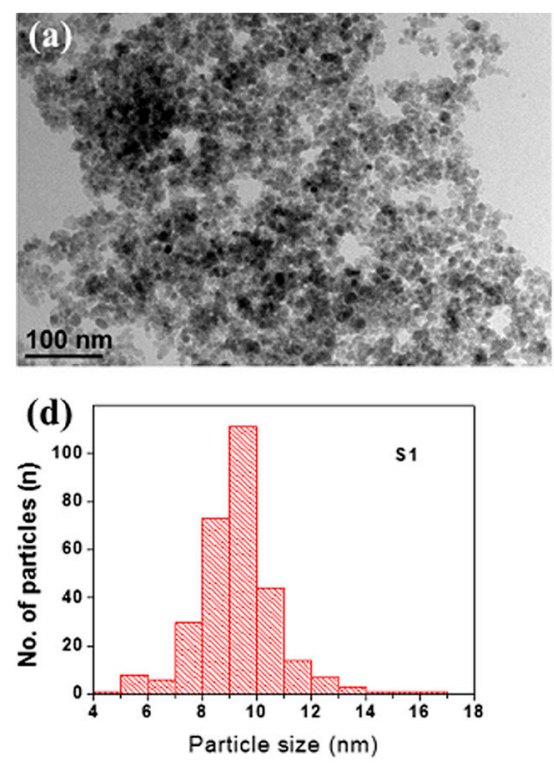
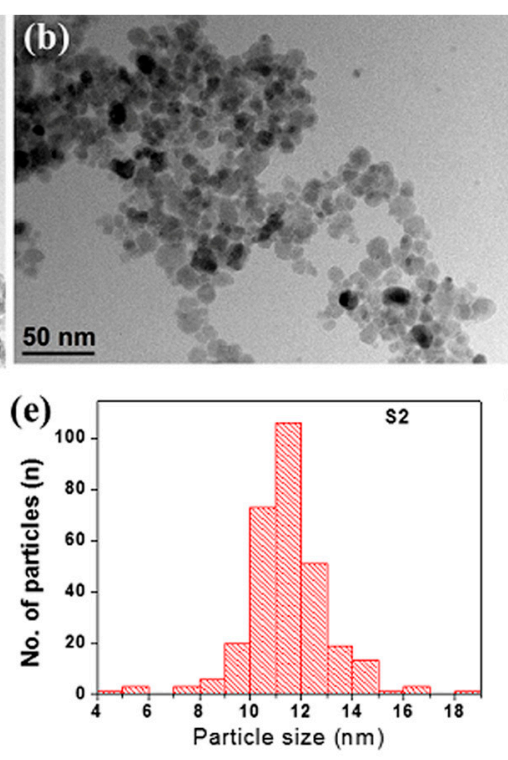
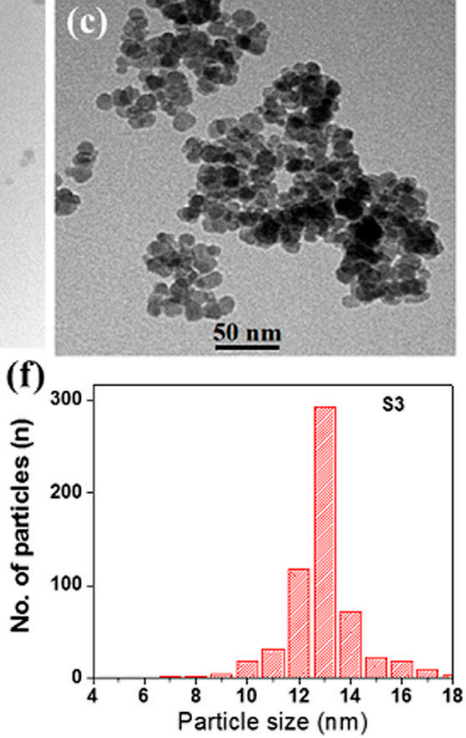

Figure 2. The transmission electron microscopy (TEM) images of samples (a) S1; (b) S2; and (c) S3. The particle size histograms of the samples (d) S1; (e) S2; and (f) S3. (Reproduced from [37]. Copyright Obaidat, I.M., et al., 2017.).

\subsection{Magnetic Properties}

Magnetization versus applied magnetic field $(M-H)$ measurements were carried out under zero field cooling (ZFC) and field cooling (FC) conditions. The measurements were conducted at several temperatures $(2,10,30,50,70$ and $100 \mathrm{~K})$ and several field cooling values, $H_{\mathrm{FC}}(0.5,1,2$ and $3 \mathrm{~T})$. In each hysteresis loop cycle, the magnetization data was recorded while the applied field was swapped between -3 to $3 \mathrm{~T}$. In the ZFC protocol, the sample was cooled down from room temperature to a particular temperature in the absence of any applied magnetic field. After that, a magnetic field was applied and ramped between $\pm \mathrm{T}$. The magnetic moment was measured during the change of the applied magnetic field. The $M-H$ hysteresis loops under ZFC at $T=2 \mathrm{~K}$, for samples S2 and S3 are shown as examples in Figure 3a,b. Typical ZFC and FC hysteresis loops were obtained for all the samples at the above mention temperatures and field cooling values. As can be seen in Figure 3, there are openings in the hysteresis loops indicating the absence of superparamagnetic behavior at $2 \mathrm{~K}$. Also, saturation magnetization was not completely achieved in both samples, even at the maximum applied field of $3 \mathrm{~T}$ (here we show up to $1 \mathrm{~T}$ only so that the openings in the hysteresis loops become visible). From these hysteresis loops we obtained the saturation magnetization, $M_{\mathrm{S}}$ at different temperatures and field cooling values, $H_{\mathrm{FC}}$.

The role of field cooling on $M_{\mathrm{S}}$ in each sample was analyzed at each temperature. The $M_{\mathrm{S}}$ for all samples did not saturate up to maximum applied field of $3 \mathrm{~T}$ at all temperatures and all $H_{\mathrm{FC}}$ values. This could be attributed to surface spin effects and inter-particle interactions. The saturation magnetization $\left(M_{\mathrm{S}}\right)$ was determined by extrapolating the high field region (at $\mathrm{H}=3 \mathrm{~T}$ ) of the $\mathrm{M}-\mathrm{H}$ curve to the infinity field. Figure 4 shows the saturation magnetization versus the shell thickness at several temperatures (from 2 to $300 \mathrm{~K}$ ) in the ZFC state as well as in the FC state at several $H_{\mathrm{FC}}$ values. Figure 5 shows the saturation magnetization versus $H_{\mathrm{FC}}$ for each sample at several temperatures (from 2 to $300 \mathrm{~K}$ ). 


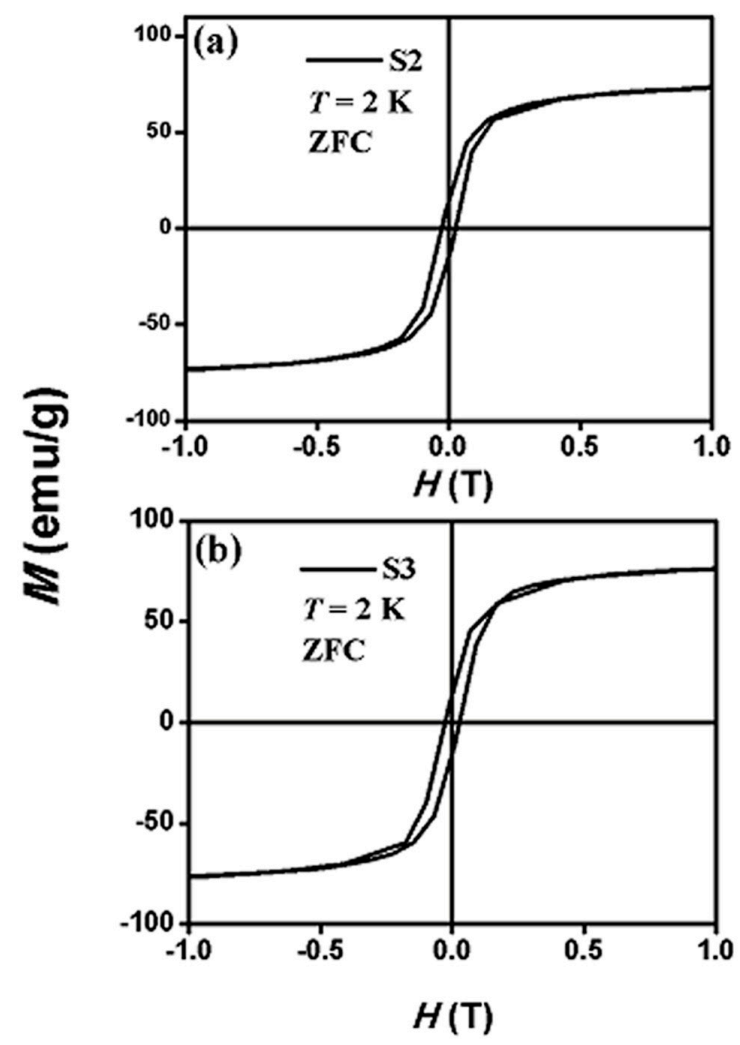

Figure 3. The applied magnetic field $(H)$ versus the magnetization $(M)$ at $2 \mathrm{~K}$ under zero field-cooling condition for (a) sample S2 and (b) sample S3. ZFC, zero field-cooling.
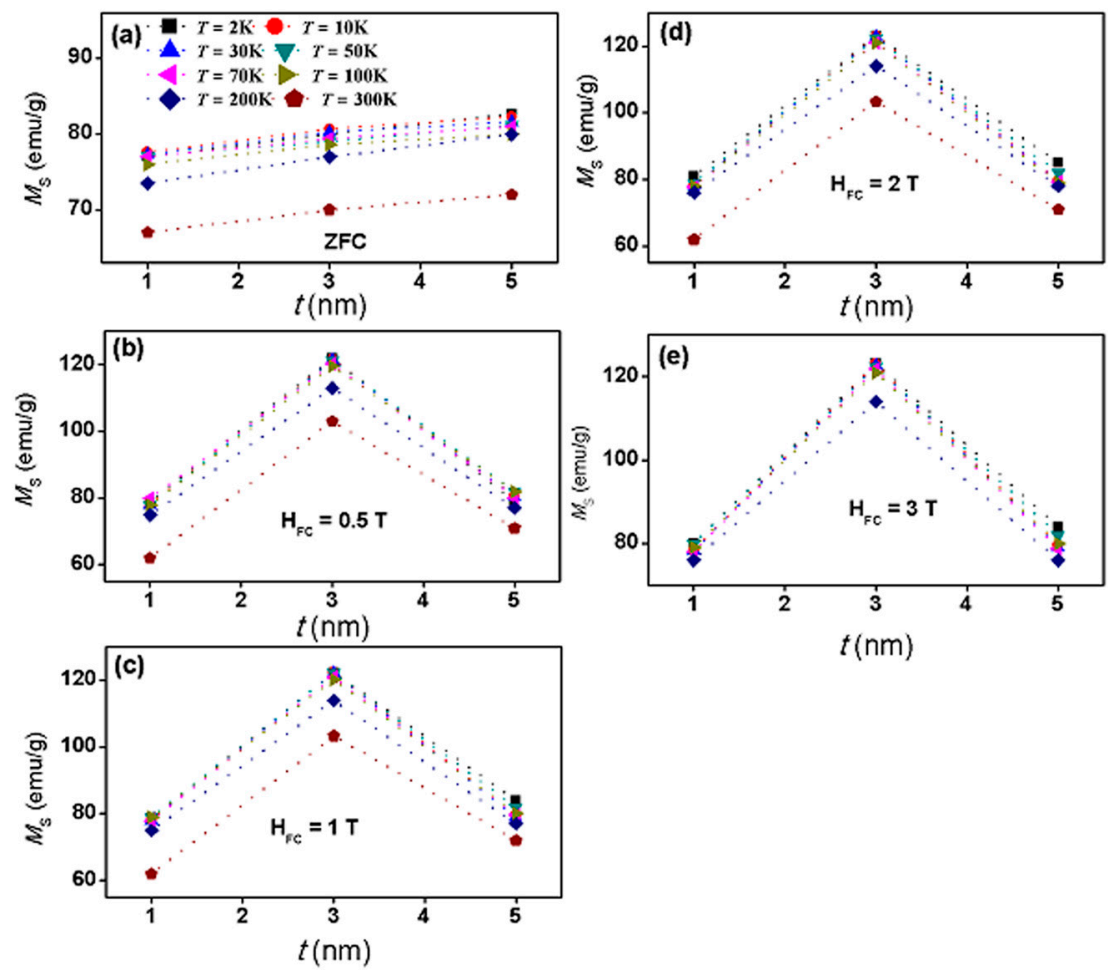

Figure 4. The saturation magnetization versus the shell thickness at several temperatures (from 2 to $300 \mathrm{~K})(\mathbf{a})$ in the ZFC state as well as in the field-cooling (FC) state at several field-cooling values of (b) $0.5 \mathrm{~T}$, (c) $1 \mathrm{~T}$, (d) $2 \mathrm{~T}$, and (e) $3 \mathrm{~T}$. 

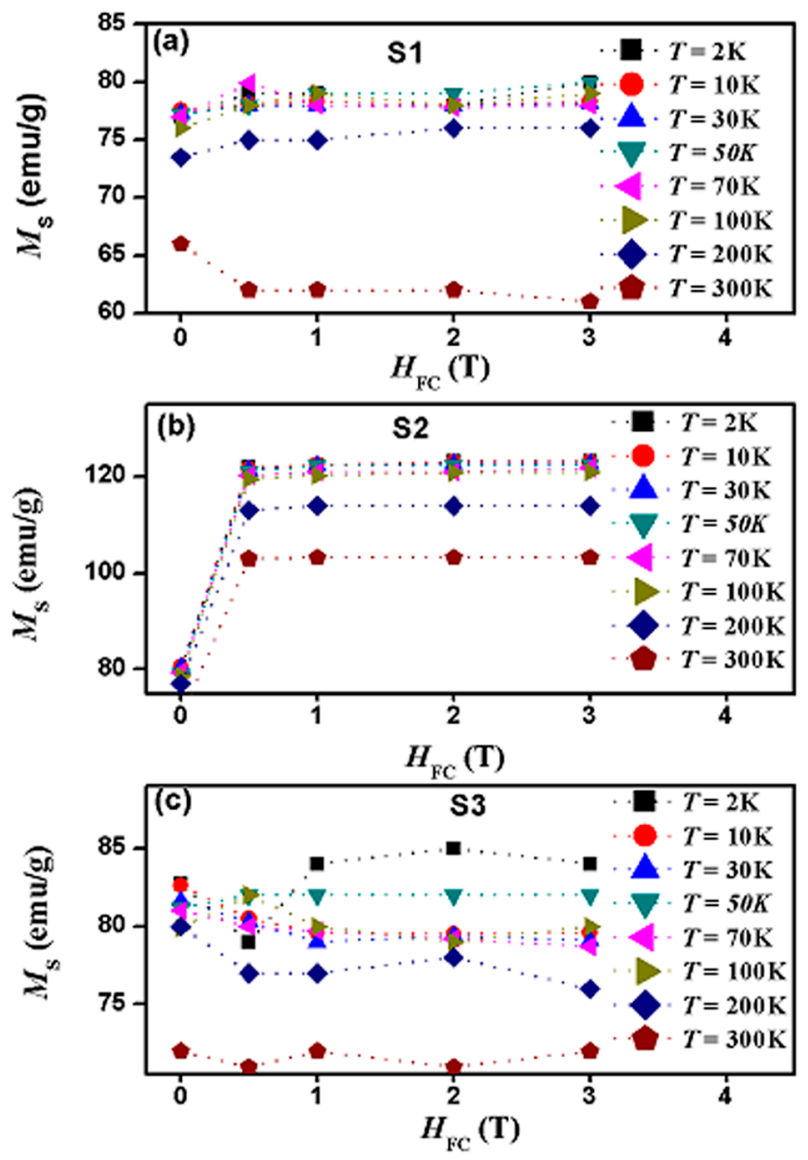

Figure 5. The saturation magnetization versus field-cooling value at several temperatures (from 2 to $300 \mathrm{~K}$ ) for each sample (a) S1, (b) S2, and (c) S3.

These two figures show some key results. (a) Saturation magnetization, with large magnitudes, has been detected in all samples at all temperatures in the ZFC state; and (b) as function of the shell thickness, in the ZFC state, the saturation magnetization has a systematic and linear increase with the increase in shell thickness at all temperatures. This reflects the significant enhancing role of shell thickens on saturation magnetization in the ZFC state. This could be due to the larger ferrimagnetic $\gamma-\mathrm{Fe}_{2} \mathrm{O}_{3}$ shell thickness (and thus volume) in sample S2. Also, this increase in $M_{\mathrm{S}}$ may be induced by the lattice strain between the $\mathrm{Fe}_{3} \mathrm{O}_{4} / \gamma-\mathrm{Fe}_{2} \mathrm{O}_{3}$ core/shell [38]. The largest values occur at $2 \mathrm{~K}$ with $77 \mathrm{emu} / \mathrm{g}$ in the sample S1, $80 \mathrm{emu} / \mathrm{g}$ in the sample S2, and a value of $84 \mathrm{emu} / \mathrm{g}$ in the sample S3; (c) shell thickness-dependent saturation magnetization values remain nearly the same with an increase in temperature from 2 to $100 \mathrm{~K}$; (d) a noticeable decrease in the shell thickness-dependent saturation magnetization values appeared above $100 \mathrm{~K}$. At $200 \mathrm{~K}$ saturation magnetization values are $73 \mathrm{emu} / \mathrm{g}$, $77 \mathrm{emu} / \mathrm{g}$ and $80 \mathrm{emu} / \mathrm{g}$ in samples S1, S2 and S3, respectively. This reflects only a decrease of less than $5 \%$ of the initial values; (e) a pronounced decrease in the shell thickness-dependent saturation magnetization values occurred at $300 \mathrm{~K}$ with minimum values of 66,69 and $72 \mathrm{emu} / \mathrm{g}$ in samples S1, S2 and S3, respectively. This reflects only a decrease of nearly $14 \%$ of the initial values. Thus, in the ZFC state, the maximum value of saturation magnetization obtained is $84 \mathrm{emu} / \mathrm{g}$ in the sample with the thickest shell (sample S3); (f) after field-cooling at $0.5 \mathrm{~T}$, the magnitudes of saturation magnetization at $2 \mathrm{~K}$ changed only slightly in samples S1 and S3 (where the saturation magnetization increased from 77 to $80 \mathrm{emu} / \mathrm{g}$ in sample S1 and decreased slightly from 84 to $80 \mathrm{emu} / \mathrm{g}$ in sample S3); (g) after field-cooling at $0.5 \mathrm{~T}$, the saturation magnetization at $2 \mathrm{~K}$ in sample S2 displays a drastic increase where it reaches $120 \mathrm{emu} / \mathrm{g}$. This indicates an increase of exactly $50 \%$ of its value in the ZFC state and a nearly $50 \%$ increase of its bulk value. This reflects the significant role of field-cooling at $0.5 \mathrm{~T}$ in enhancing the 
saturation magnetization in sample S2. This could be attributed to the enhancement of the alignment of both the core/shell interface and the surface spins; (h) after field-cooling at $0.5 \mathrm{~T}$, the magnitudes of saturation magnetization at temperatures up to $100 \mathrm{~K}$ remained almost equal to those at $2 \mathrm{~K}$ in all samples. However, a noticeable decrease of the saturation magnetization values occurs at 200 and $300 \mathrm{~K}$ in all samples. For example, in S2 the saturation magnetization decreased to $115 \mathrm{emu} / \mathrm{g}$ at $200 \mathrm{~K}$ and to $105 \mathrm{emu} / \mathrm{g}$ at $300 \mathrm{~K}$, which is much larger than the maximum value obtained in the ZFC state ( $80 \mathrm{emu} / \mathrm{g}$ at $2 \mathrm{~K}$ ). Interestingly, in sample S2, all the saturation magnetization values in the FC state are nearly $50 \%$ larger than the values in the ZFC state at similar temperatures. This clearly reflects the significant role of field-cooling at $0.5 \mathrm{~T}$ in enhancing the saturation magnetization in sample S2 even at room temperature; (i) larger field-cooling values displayed similar effect as that of the $0.5 \mathrm{~T}$ field-cooling value. This reflects the ineffective role of field-cooling above $0.5 \mathrm{~T}$ in enhancing the saturation magnetization in all samples; ( $\mathrm{j}$ ) thus, the $0.5 \mathrm{~T}$ can be considered as a critical field-cooling value in samples S2 where a drastic increase in saturation magnetization occurred; $(\mathrm{k})$ this also shows that the shell thickness of $3 \mathrm{~nm}$ is a critical thickness in this core/shell nanoparticle system where a very large enhancement in saturation magnetization occurred at the field-cooling value of $0.5 \mathrm{~T}$ at all temperatures with a value of $120 \mathrm{emu} / \mathrm{g}$ at temperatures up to $100 \mathrm{~K}$ and with only slight decrease at temperatures above $100 \mathrm{~K}$.

The temperature dependence of the saturation magnetization, $M_{\mathrm{S}}$ in bulk ferromagnetic or ferrimagnetic materials at low temperatures is governed by the Bloch's law [39-42].

$$
M(T)=M_{0}\left[1-\left(\frac{T}{T_{0}}\right)^{\alpha_{B}}\right] \ldots
$$

where, $M_{0}$ is the saturation magnetization at $0 \mathrm{~K}, T_{0}$ is the temperature at which the $M_{\mathrm{S}}$ becomes zero (ferromagnetic transition temperature, $T_{C}$ ). The exponent $\alpha_{B}$ is known as the Bloch's exponent $\left(\alpha_{B}\right)$ and the value of $\alpha_{B}$ is $3 / 2$ for bulk materials. Bloch proposed the $T^{3 / 2}$ law by considering the magnon excitation of long wave-length spin-waves at low temperatures [39].

Figure 6 shows the temperature dependence of $M_{\mathrm{S}}$ measured under the ZFC protocol for all the samples. We found that the experimental $M_{\mathrm{S}}$ values of our core/shell nanoparticles deviate from original Bloch's law. Hence, we have fitted the experimental data to a modified Bloch's law for each sample. The fitted data for each sample is shown as a solid line in Figure 6. The experimental data was fitted using Equation (1) and the fitted magnetic parameters are shown in Table 1. For nanoparticles, the finite size effects are expected to be significant resulting in deviations from the ideal Bloch's equation. The values of $\alpha_{B}$ for nanoparticles were reported to vary within the range of 1.5 to 2.0 [43-48]. In our samples, it is observed from the fitting parameters that the modified exponent $\left(\alpha_{B}\right)$ is larger than the exponent for bulk materials. The values of the modified Bloch exponent inferred from the fitting data for S1, S2 and S3 samples are 2.0, 2.5 and 3.0, respectively. As can be seen in Table 1, $\alpha_{B}$ increases monotonically (and linearly) with shell thickness. This indicates that the larger the shell thickness, the larger is the deviation from the original Bloch's law. For normal nanoparticles, it is expected that as the size of the particle increases, $\alpha_{B}$ should approach that of the bulk materials. However, for our core/shell structured nanoparticles, the surface and interface have contributed effectively such that the opposite behavior is obtained. The deviation from the original Bloch's law could be attributed to the surface and interface effects [49-52]. Due to the broken exchange bonds [53] at the core/shell interface, the interface spins are suggested to be in disordered states. We suggest that (as shown in [37]) at the core/shell interface, spin-glass like structures $[49,50]$ occur with amounts that increase with shell thickness. Hence, we attribute the increase of $\alpha_{B}$ (increase of deviation from the original Bloch's law) with shell thickness to the increase of spin-glass structures as the shell thickness increases. The surface spins also could contribute to this deviation where the role of surface spins increases with decreasing the total size of the particle. 

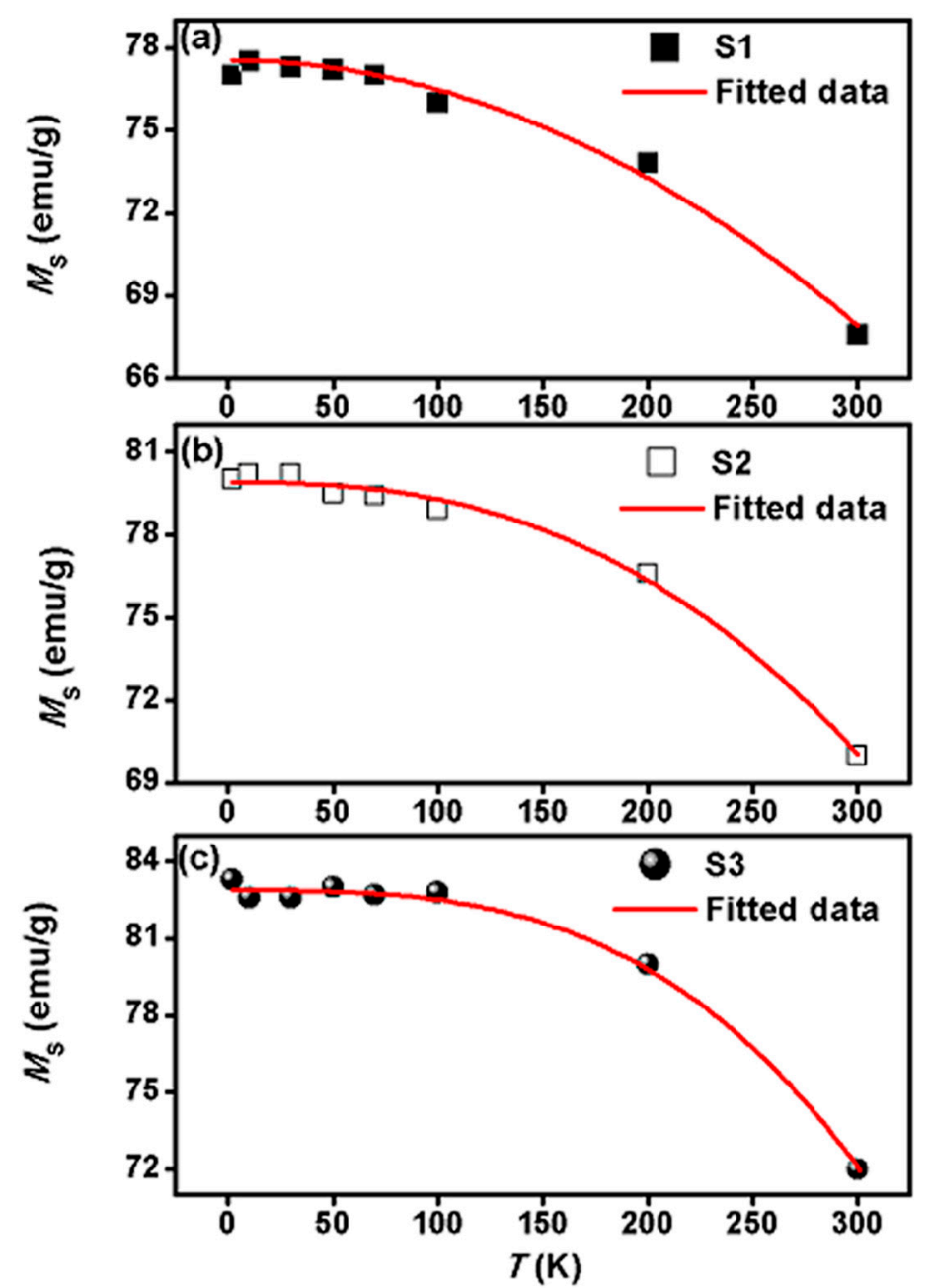

Figure 6. The temperature (T) dependent $M_{\mathrm{S}}$ for samples (a) S1; (b) S2; and (c) S3, under zero field-cooling condition. The solid lines represent the fittings of the experimental data to a modified Bloch's law.

Table 1. The fitting parameters of the experimental data to a modified Bloch's law ${ }^{1}$.

\begin{tabular}{ccccc}
\hline \multirow{2}{*}{ Sample } & \multirow{2}{*}{ Shell Thickness $(\mathbf{n m})$} & \multicolumn{2}{c}{ Fitted Parameters at ZFC } & \multirow{2}{*}{$\boldsymbol{\alpha B}$} \\
\cline { 3 - 4 } & & $\boldsymbol{M}_{\mathbf{0}} \mathbf{( e m u / g )}$ & $\boldsymbol{T}_{\mathbf{0}} \mathbf{( K )}$ & \\
\hline S1 & 1 & $77.5( \pm 0.4)$ & $852( \pm 15)$ & 2.0 \\
S2 & 3 & $79.9( \pm 0.1)$ & $689( \pm 10)$ & 2.5 \\
S3 & 5 & $82.8( \pm 0.2)$ & $580( \pm 5)$ & 3.0 \\
\hline \multicolumn{4}{c}{}
\end{tabular}

The role of field cooling on $M_{S}(T)$ was investigated on all samples. Here we present the results on the sample S1. Typical results were obtained on the other samples. Figure 7 shows the temperature dependence of $M_{\mathrm{S}}$ in sample $\mathrm{S} 1$. The $M_{\mathrm{S}}$ values were obtained from the magnetic hysteresis measurements under both the ZFC and the FC protocols at the field cooling values: $H_{\mathrm{FC}}=0.5,1,2$ and $3 \mathrm{~T}$. We found that the experimental $M_{\mathrm{S}}$ values for our core/shell sample in all conditions deviate considerably from the original Bloch's law. Thus, we fitted the temperature dependence of $M_{\mathrm{S}}$ in each case to a modified Bloch's law (shown as dashed lines in Figure 7) by varying the parameters $M_{0}, T_{0}$ and $\alpha_{B}$. For the ZFC state, $\alpha_{B}$ was found to be nearly 3.1, which is a large deviation from the value of 1.5 for the original Bloch's law. 

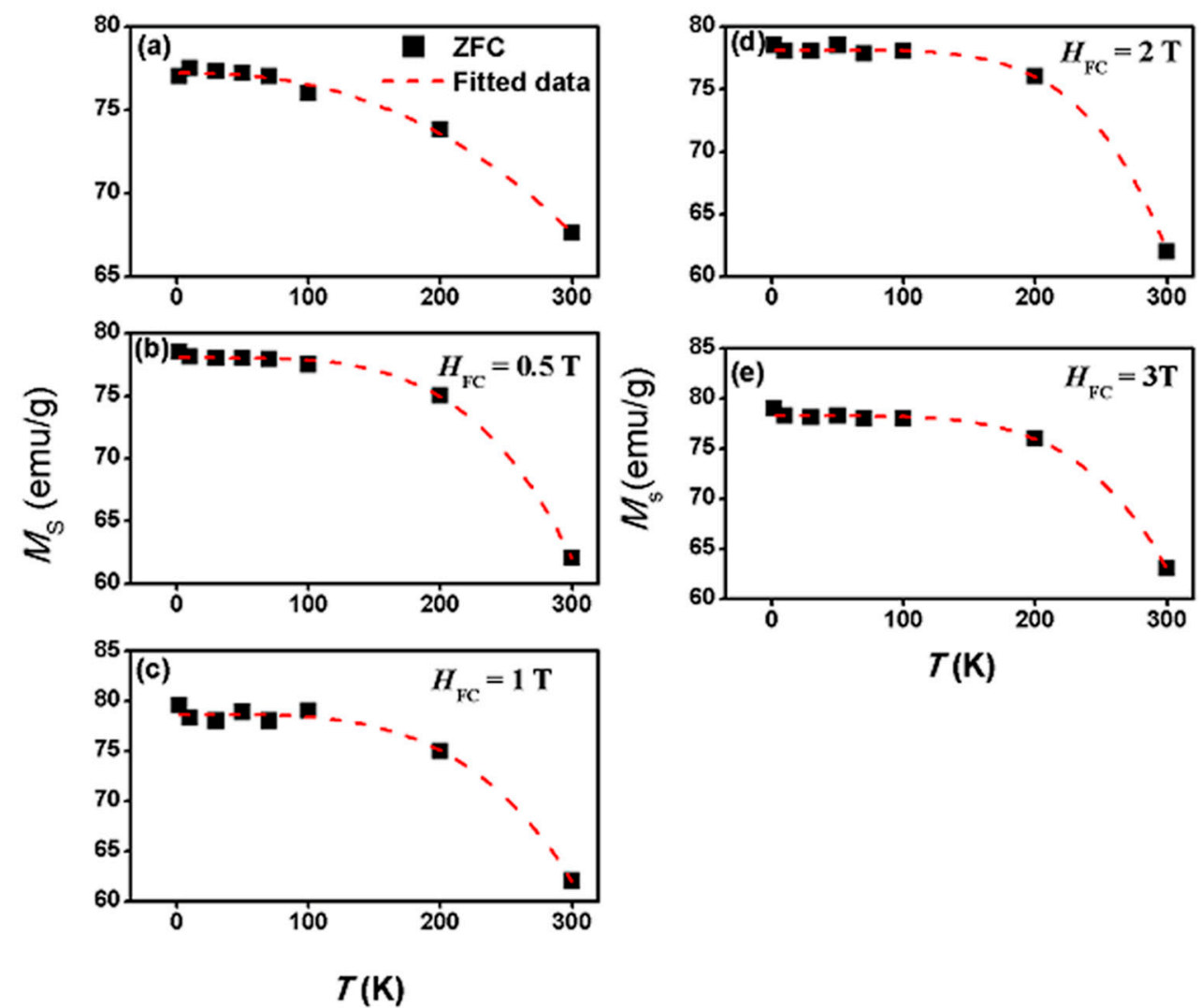

Figure 7. Temperature dependence of the saturation magnetization (in sample S1) measured under (a) the zero field-cooling (ZFC) condition and under the field-cooling (FC) condition at several field-cooling values of (b) $0.5 \mathrm{~T}$, (c) $1 \mathrm{~T}$, (d) $2 \mathrm{~T}$, and (e) $3 \mathrm{~T}$. The dashed lines represent the fittings of the experimental data to modified Bloch's laws.

Field-cooling shows a significant effect on $\alpha_{B}$ where the values are considerably larger than that for the ZFC state (displayed in Table 2) and thus reflect the significant role of field-cooling on $M_{\mathrm{S}}(T)$.

Table 2. The fitting parameters of the experimental data to a modified Bloch's law in sample S1.

\begin{tabular}{cccc}
\hline \multirow{2}{*}{$\boldsymbol{H}_{\mathrm{FC}}(\mathrm{T})$} & \multicolumn{2}{c}{ Fitted Parameters } & \multirow{2}{*}{$\boldsymbol{\alpha}_{\boldsymbol{B}}$} \\
\cline { 2 - 3 } & $\boldsymbol{M}_{\mathbf{0}}(\mathbf{e m u} / \mathbf{g})$ & $\boldsymbol{T}_{\mathrm{C}}(\mathbf{K})$ & \\
\hline 0.5 & $78.0 \pm 0.1$ & $443 \pm 5$ & 4.0 \\
1 & $78.7 \pm 0.14$ & $452 \pm 2.6$ & 3.8 \\
2 & $78.1 \pm 0.6$ & $412 \pm 9.9$ & 4.9 \\
3 & $78.3 \pm 0.2$ & $427 \pm 3$ & 4.6 \\
\hline
\end{tabular}

The $M_{S}(T)$ for nanoparticles were reported to deviate from the Bloch's law for bulk materials where the Bloch's exponent $\alpha_{B}$ could have values larger or smaller than $3 / 2$ [46,54,55]. Various suggestions were made to explain the deviation from the Bloch's law in nanoparticle systems [49,56-62]. For nickel ferrite nanoparticles, the modified Bloch's model was found to be valid for temperatures between 50 and $300 \mathrm{~K}$ [39]. At temperatures lower than $50 \mathrm{~K}$, the authors reported a deviation from the modified Bloch law where a sharp increase in saturation magnetization occurred [39]. This anomaly was suggested to be due to some extra contribution of the shell-spins moment to the total magnetization at low temperatures $[39,60]$ or due to the presence of some paramagnetic impurities in the nanoparticles which would be activated at low temperatures [39,61]. 
The effect of core/shell on different magnetic properties has been reported in many studies [30-35]. However, we are not aware of a research study on the effect of shell thickness and field cooling on $M_{\mathrm{S}}$ and $M_{\mathrm{S}}(T)$. In our system of $\mathrm{Fe}_{3} \mathrm{O}_{4} / \gamma-\mathrm{Fe}_{2} \mathrm{O}_{3}$ core/shell nanoparticles, it is very evident that the shell thickness and field cooling have significant influence on $M_{\mathrm{S}}$ and $M_{\mathrm{S}}(T)$. It is observed that as the shell thickness increases, the temperature dependence of saturation magnetization deviates nonmonotonically from the original Bloch's law and the deviation is maximum for the nanoparticle with shell thickness of $5 \mathrm{~nm}$. These results could be attributed to the existence of interface spin-glass clusters (SGCs) which occurred due to structural defects at the core/shell interface. The existence of such (SGCs) was investigated and reported in our recently published paper [37]. The spins in these SGCs are not aligned in a particular direction due to different spin interactions among the spins of each cluster and the interactions with the core and shell spins. In our core/shell particle system, we suggest that, as the shell thickness varies, the SGCs with randomly oriented spins vary, leading to a varied magnetic anisotropy at the interface and hence resulting in deviations from the original Bloch's law.

\section{Conclusions}

We have successfully synthesized $\mathrm{Fe}_{3} \mathrm{O}_{4} / \gamma-\mathrm{Fe}_{2} \mathrm{O}_{3}$ core/shell nanoparticles with nearly fixed core diameter and varied shell thicknesses. The core dimensions of all the samples were approximately $8 \mathrm{~nm}$ and the shell thicknesses were varied to $1 \mathrm{~nm}, 3 \mathrm{~nm}$ and $5 \mathrm{~nm}$, for samples S1, S2 and S3, respectively. The role of field shell thickness and field cooling on the temperature dependence of saturation magnetization was revealed where significant deviations from the original Bloch's law were obtained. The shell thickness was found to have a considerable impact on the magnitude of the saturation magnetization. The role of field cooling on the magnitude of the saturation magnetization was found to be very significant in sample S2. These results are attributed to modifications in the spin-glass clusters at the interface which lead to variations in the interfacial magnetic anisotropy.

Acknowledgments: This work was financially supported by the UAEU Program for Advanced Research (UPAR) under the Grant No. 31S162.

Author Contributions: Ihab M. Obaidat conceived the ideas and designed the experiments; Chiranjib Nayek synthesized the samples; Chiranjib Nayek carried out the XRD and other measurements; Kaustuv Manna conducted the magnetization measurements; Chiranjib Nayek and Ihab M. Obaidat analyzed the data; Ihab M. Obaidat explained and discussed and the findings; Ihab M. Obaidat wrote the manuscript.

Conflicts of Interest: The authors declare no conflict of interest.

\section{References}

1. Gubin, S.P.; Koksharov, Y.A.; Khomutov, G.B.; Yurkov, G.Y. Magnetic nanoparticles: Preparation, structure and properties. Russ. Chem. Rev. 2005, 74, 489-520. [CrossRef]

2. Obaidat, I.M.; Issa, B.; Haik, Y. The role of aggregation of ferrite nanoparticles on their magnetic properties. J. Nanosci. Nanotechnol. 2011, 11, 3882-3889. [CrossRef] [PubMed]

3. Lubbe, A.S.; Bergmann, C.; Riess, H.; Schriver, F.; Reichardt, P.; Possinger, K.; Matthias, M.; Dorken, B.; Herrmann, F.; Gürtler, R.; et al. Clinical experiences with magnetic drug targeting: A phase I study with $4^{\prime}$-epidoxorubicin in 14 patients with advanced solid tumors. Cancer Res. 1996, 56, 4686-4693. [PubMed]

4. Larsen, B.A.; Haag, M.A.; Serkova, N.J.; Shroyer, K.R.; Stoldt, C.R. Controlled aggregation of superparamagnetic iron oxide nanoparticles for the development of molecular magnetic resonance imaging probes. Nanotechnology 2008, 19, 265102-265108. [CrossRef] [PubMed]

5. Issa, B.; Obaidat, I.M.; Albiss, B.A.; Haik, Y. Magnetic nanoparticles: Surface effects and properties related to biomedicine applications. Int. J. Mol. Sci. 2013, 14, 21266-21305. [CrossRef] [PubMed]

6. Obaidat, I.M.; Issa, B.; Haik, Y. Magnetic properties of magnetic nanoparticles for efficient hyperthermia. Nanomaterials 2015, 5, 63-89. [CrossRef] [PubMed]

7. Zhu, D.; Liu, F.; Ma, L.; Liu, D.; Wang, Z. Nanoparticle-based systems for $T_{1}$-weighted magnetic resonance imaging contrast agents. Int. J. Mol. Sci. 2013, 14, 10591-10607. [CrossRef] [PubMed] 
8. Ito, A.; Honda, H.; Kobayashi, T. Cancer immunotherapy based on intracellular hyperthermia using magnetite nanoparticles: A novel concept of "heat-controlled necrosis" with heat shock protein expression. Cancer Immunol. Immunother. 2006, 55, 320-328. [CrossRef] [PubMed]

9. Ammar, S.; Jouini, N.; Fievet, F.; Stephan, O.; Marhic, C.; Richard, M.; Villain, F.; Chartier dit Moulin, C.; Brice, S.; Sainctavit, P. Influence of the synthesis parameters on the cationic distribution of $\mathrm{ZnFe}_{2} \mathrm{O}_{4}$ nanoparticles obtained by forced hydrolysis in polyol medium. J. Non-Cryst. Solids 2004, 345, 658-662. [CrossRef]

10. Sato, T.; Haneda, K.; Seki, M.; Iijima, T. Morphology and magnetic properties of ultrafine $\mathrm{ZnFe}_{2} \mathrm{O}_{4}$ particles. Appl. Phys. A 1990, 50, 13-16. [CrossRef]

11. Kamiyama, T.; Haneda, K.; Sato, T.; Ikeda, S.; Asano, H. Cation distribution in $\mathrm{ZnFe}_{2} \mathrm{O}_{4}$ fine particles studied by neutron powder diffraction. Solid State Commun. 1992, 81, 563-566. [CrossRef]

12. Jeyadevan, B.; Tohji, K.; Nakatsuka, K. Structure analysis of coprecipitated $\mathrm{ZnFe}_{2} \mathrm{O}_{4}$ by extended X-ray-absorption fine structure. Appl. Phys. 1994, 76, 6325-6327. [CrossRef]

13. Hamdeh, H.H.; Ho, J.C.; Oliver, S.A.; Willey, R.J.; Oliveri, G.; Busca, G. Magnetic properties of partially-inverted zinc ferrite aerogel powders. J. Appl. Phys. 1997, 81, 1851-1857. [CrossRef]

14. Makovec, D.; Kodre, A.; Arčon, I.; Drofenik, M. Structure of manganese zinc ferrite spinel nanoparticles prepared with co-precipitation in reversed microemulsions. J. Nanopart. Res. 2009, 11, 1145-1158. [CrossRef]

15. Makovec, D.; Kodre, A.; Arčon, I.; Drofenik, M. The structure of compositionally constrained zinc-ferrite spinel nanoparticles. J. Nanopart. Res. 2011, 13, 1781-1790. [CrossRef]

16. Kodama, R.H.; Berkowitz, A.E.; McNiff, E.J., Jr.; Foner, S. Surface spin disorder in $\mathrm{NiFe}_{2} \mathrm{O}_{4}$ nanoparticles. Phys. Rev. Lett. 1996, 77, 394-397. [CrossRef] [PubMed]

17. Kodama, R.H.; Berkowitz, A.E.; McNiff, E.J., Jr.; Foner, S. Surface spin disorder in ferrite nanoparticles. J. Appl. Phys. 1997, 81, 5552-5557. [CrossRef]

18. Kodama, R.H.; Berkowitz, A.E. Atomic-scale magnetic modeling of oxide nanoparticles. Phys. Rev. B 1999, 59, 6321-6336. [CrossRef]

19. Gazeau, F.; Dubois, E.; Hennion, M.; Perzynski, R.; Raikher, Y. Quasi-elastic neutron scattering on $\gamma-\mathrm{Fe}_{2} \mathrm{O}_{3}$ nanoparticles. Europhys. Lett. 1997, 40, 575-580. [CrossRef]

20. Hennion, M.; Bellouard, C.; Mirebeau, I.; Dormann, J.L.; Nogues, M. Dual spin dynamics of small fe particles. Europhys. Lett. 1994, 25, 43-48. [CrossRef]

21. Bellouard, C.; Mirebeau, I.; Hennion, M. Magnetic correlations of fine ferromagnetic particles studied by small-angle neutron scattering. Phys. Rev. B 1996, 53, 5570-5578. [CrossRef]

22. Rosensweig, R.E. Heating magnetic fluid with alternating magnetic field. J. Magn. Magn. Mater. 2002, 252, 370-374. [CrossRef]

23. Ondeck, C.L.; Habib, A.H.; Ohodnicki, P.; Miller, K.; Sawyer, C.A.; Chaudhary, P.; McHenry, M.E. Theory of magnetic fluid heating with an alternating magnetic field with temperature dependent materials properties for self-regulated heating. J. Appl. Phys. 2009, 105, 07B324. [CrossRef]

24. Nayek, C.; Manna, K.; Bhattacharjee, G.; Murugavel, P.; Obaidat, I.M. Investigating size- and temperature-dependent coercivity and saturation magnetization in PEG coated $\mathrm{Fe}_{3} \mathrm{O}_{4}$ nanoparticles. Magnetochemistry 2017, 3, 19. [CrossRef]

25. Obaidat, I.M.; Issa, B.; Albiss, B.A.; Haik, Y. Temperature dependence of saturation magnetization and coercivity in $\mathrm{Mn}_{0.5} \mathrm{Zn}_{0.5} \mathrm{Gd}_{0.02} \mathrm{Fe}_{1.98} \mathrm{O}_{4}$ ferrite nanoparticles. IOP Conf. Ser. Mater. Sci. Eng. 2015, $92,012012$. [CrossRef]

26. Nairan, A.; Khan, M.; Khan, U.; Iqbal, M.; Riaz, S.; Naseem, S. Temperature-dependent magnetic response of antiferromagnetic doping in cobalt ferrite nanostructures. Nanomaterials 2017, 6, 73. [CrossRef] [PubMed]

27. Sahu, B.N.; Doshi, A.S.; Prabhu, R.; Venkataramani, N.; Prasad, S.; Krishnan, R. Temperature dependence of FMR and magnetization in nanocrystalline zinc ferrite thin films. AIP Adv. 2016, 6, 055928. [CrossRef]

28. Khajonrit, J.; Wongpratat, U.; Kidkhunthod, P.; Pinitsoontorn, S.; Maensiri, S. Effects of Co doping on magnetic and electrochemical properties of $\mathrm{BiFeO}_{3}$ nanoparticles. J. Magn. Magn. Mater. 2018, 449, $423-434$. [CrossRef]

29. Choo, S.; Lee, K.; Jo, Y.; Yoon, S.; Choi, J.; Kim, J.; Park, J.; Lee, K.; Lee, J.; Jung, M. Interface effect of magnetic properties in Ni nanoparticles with a hcp core and fcc shell structure. Nanosci. Nanotechnol. 2011, 11, 6126-6130. [CrossRef] 
30. Luis, F.S.; Bartolome, F.; Petroff, F.; Bartolome, J.; Garcia, M.; Deranlot, C.; Jaffres, M.; Martinez, H.; Bencok, P.; Wilhelm, F.; et al. Tuning the magnetic anisotropy of Co nanoparticles by metal capping. Europhys. Lett. 2006, 76, 142-148. [CrossRef]

31. Skomski, R.; Coey, J.M.D. Giant energy product in nanostructured two-phase magnets. Phys. Rev. B 1993, 48, 15812-15816. [CrossRef]

32. Fullerton, E.E.; Jiang, J.S.; Bader, S.D. Hard/soft magnetic heterostructures: Model exchange-spring magnets. J. Magn. Magn. Mater. 1999, 200, 392-404. [CrossRef]

33. Zeng, H.; Sun, S.; Li, J.; Wang, Z.L.; Liu, J.P. Tailoring magnetic properties of core/shell nanoparticles. Appl. Phys. Lett. 2004, 85, 792-794. [CrossRef]

34. Zeng, H.; Li, J.; Liu, J.P.; Wang, Z.L.; Sun, S. Exchange-coupled nanocomposite magnets by nanoparticle self-assembly. Nature 2002, 420, 395-398. [CrossRef] [PubMed]

35. Qiang, Y.; Antony, J.; Sharma, A.; Nutting, J.; Sikes, D.; Meyer, D. Iron/iron oxide core-shell nanoclusters for biomedical applications. J. Nanopart. Res. 2006, 8, 489-496. [CrossRef]

36. Grosvenor, A.P.; Kobe, B.A.; McIntyre, N.S. Examination of the oxidation of iron by oxygen using X-ray photoelectron spectroscopy and QUASESTM . Surf. Sci. 2004, 565, 151-162. [CrossRef]

37. Obaidat, I.M.; Nayek, C.; Manna, K.; Bhattacharya, G.; Al-Omari, I.; Gismelseed, A. Investigating exchange bias and coercivity in $\mathrm{Fe}_{3} \mathrm{O}_{4} / \gamma-\mathrm{Fe}_{2} \mathrm{O}_{3}$ core/shell nanoparticles of fixed core diameter and variable shell thicknesses. Nanomaterials 2017, 7, 415. [CrossRef] [PubMed]

38. Zhou, S.M.; Imhoff, D.; Yu-Zhang, K.; Leprince-Wang, Y. Effect of field cooling on magnetic properties of ultrafine CoO/Co particles. Appl. Phys. A Mater. Sci. Process. 2005, 81, 115-118. [CrossRef]

39. Maaz, K.; Mumtaz, A.; Hasanain, S.K.; Bertino, M.F. Temperature dependent coercivity and magnetization of nickel ferrite nanoparticles. J. Magn. Magn. Mater. 2010, 322, 2199-2202. [CrossRef]

40. Kneller, E.F.; Luborsky, F.E. Particle size dependence of coercivity and remanence of single-domain particles. J. Appl. Phys. 1963, 34, 656-658. [CrossRef]

41. Batlle, X.; Garcia del Muro, M.; Tejada, J.; Pfeiffer, H.; Gornert, P.; Sinn, E. Magnetic study of M-type doped barium ferrite nanocrystalline powders. J. Appl. Phys. 1993, 74, 3333-3340. [CrossRef]

42. Bloch, F. Zur theorie des ferromagnetismus. Z. Phys. 1930, 61, 206-219. [CrossRef]

43. Torre, E.D.; Bennett, L.H.; Watson, R.E. Extension of the Bloch $T^{3 / 2}$ law to magnetic nanostructures: Bose-Einstein condensation. Phys. Rev. Lett. 2005, 94, 147210-147212. [CrossRef] [PubMed]

44. Senz, V.; Röhlsberger, R.; Bansmann, J.; Leupold, O.; Meiwes-Broer, K.-H. Temperature dependence of the magnetization in Fe islands on W(110): Evidence for spin-wave quantization. New J. Phys. 2003, 5, 47. [CrossRef]

45. Hendriksen, P.V.; Linderoth, S.; Lindgard, P.A. Finite-size effects in the magnetic properties of ferromagnetic clusters. J. Magn. Magn. Mater. 1992, 104-107, 1577-1579. [CrossRef]

46. Hendriksen, P.V.; Linderoth, S.; Lindgard, P.A. Finite-size modifications of the magnetic properties of clusters. Phys. Rev. B 1993, 48, 7259-7273. [CrossRef]

47. Linderoth, S.; Balcells, L.; Labarta, A.; Tejada, J.; Hendriksen, P.V.; Sethi, S.A. Magnetization and mossbauer studies of ultrafine Fe-C particles. J. Magn. Magn. Mater. 1993, 124, 269-276. [CrossRef]

48. Eggeman, A.S.; Petford-Long, A.K.; Dobson, P.J.; Wiggins, J.; Bromwich, T.; Dunin-Borkowski, R.; Kasama, T. Synthesis and characterisation of silica encapsulated cobalt nanoparticles and nanoparticle chains. J. Magn. Magn. Mater. 2006, 301, 336-342. [CrossRef]

49. Del Bianco, L.; Fiorani, D.; Testa, A.M.; Bonetti, E. Exchange bias in the nanogranular Fe/Feoxide system. J. Magn. Magn. Mater. 2005, 290-291, 102-105. [CrossRef]

50. Nogues, J.; Sort, J.; Langlais, V.; Skumryev, V.; Surinach, S.; Munoz, J.S.; Baro, M.D. Exchange bias in nanostructures. Phys. Rep. 2015, 422, 65-117. [CrossRef]

51. Kommareddi, N.S.; Tata, M.; John, V.T.; McPherson, G.L.; Herman, M.F.; Lee, Y.-S.; O'Connor, C.J.; Akkara, J.A.; Kaplan, D.L. Synthesis of superparamagnetic polymer-ferrite composites using surfactant microstructures. Chem. Mater. 1996, 8, 801-809. [CrossRef]

52. Morup, S.; Topsoe, H.J. Mössbauer studies of thermal excitations in magnetically ordered microcrystals. Appl. Phys. 1976, 11, 63-66. [CrossRef]

53. Abdallah, H.M.I.; Moyo, T.; Ezekiel, I.P.; Osman, N.S.E. Structural and magnetic properties of $\mathrm{Sr}_{0.5} \mathrm{Co}_{0.5} \mathrm{Fe}_{2} \mathrm{O}_{4}$ nanoferrite. J. Magn. Magn. Mater. 2014, 365, 83-87. [CrossRef] 
54. Ortega, D.; Vélez-Fort, E.; García, D.A.; García, R.; Litrán, R.; Barrera-Solano, C.; Ramírez-del-Solar, M.; Domínguez, M. Size and surface effects in the magnetic properties of maghemite and magnetite coated nanoparticles. Philos. Trans. R. Soc. A 2010, 368, 4407-4418. [CrossRef] [PubMed]

55. Gubin, S.P. (Ed.) Magnetic Nanoparticles; Wiley-VCH: Berlin, Germany, 2009; ISBN 978-3-527-40790-3.

56. Alves, C.R.; Aquino, R.; Sousa, M.H.; Rechenberg, H.R.; Goya, G.F.; Tourinho, F.A.; Depeyrot, J. Low temperature experimental investigation of finite-size and surface effects in $\mathrm{CuFe}_{2} \mathrm{O}_{4}$ nanoparticles of ferrofluids. J. Metastab. Nanocryst. Mater. 2004, 20-21, 694-699. [CrossRef]

57. Mandal, K.; Mitra, S.; Kumar, P.A. Deviation from Bloch $T^{3 / 2}$ law in ferrite nanoparticles. Europhys. Lett. 2006, 75, 618-623. [CrossRef]

58. Kumar, P.A.; Mandal, K. Effect of spatial confinement on spin-wave spectrum: Low temperature deviation from Bloch $T^{3 / 2}$ law in Co nanoparticles. arXiv 2007, arXiv:cond-mat/0701152.

59. Mathur, P.; Thakur, A.; Singh, M. Effect of nanoparticles on the magnetic properties of Mn-Zn soft ferrite. J. Magn. Magn. Mater. 2008, 320, 1364-1369. [CrossRef]

60. Tung, L.D.; Kolesnichenko, V.; Caruntu, D.; Chou, N.H.; O'Connor, C.J.; Spinu, L. Magnetic properties of ultrafine cobalt ferrite particles. J. Appl. Phys. 2016, 93, 7486-7488. [CrossRef]

61. Morup, S. Comment on “Deviation from the Bloch $T^{3 / 2}$ law in ferrite nanoparticles" by K. Mandal et al. Europhys. Lett. 2007, 77, 27003. [CrossRef]

62. Hajra, P.; Basu, S.; Dutta, S.; Brahma, P.; Chakravorty, D. Exchange bias in ferrimagnetic-antiferromagnetic nanocomposite produced by mechanical attrition. J. Magn. Magn. Mater. 2009, 321, 2269-2275. [CrossRef]

(C) 2017 by the authors. Licensee MDPI, Basel, Switzerland. This article is an open access article distributed under the terms and conditions of the Creative Commons Attribution (CC BY) license (http:/ / creativecommons.org/licenses/by/4.0/). 